PROCEEDINGS OF THE

AMERICAN MATHEMATICAL SOCIETY

Volume 125, Number 8, August 1997, Pages 2239-2240

S 0002-9939(97)03820-3

\title{
ARTINIAN RIGHT SERIAL RINGS
}

\author{
SURJEET SINGH \\ (Communicated by Ken Goodearl)
}

\begin{abstract}
Let $R$ be an artinian ring such that for the Jacobson radical $J$ of $R$, $R / J$ is a direct product of matrix rings over finite-dimensional division rings. Then the following are proved to be equivalent: (1) Every indecomposable injective left $R$-module is uniserial. (2) $R$ is right serial.
\end{abstract}

A module with the lattice of its submodules linearly ordered under inclusion, is called a uniserial module. A ring $R$ is left (right) serial, if it is a direct sum of uniserial left (right) modules. Let $R$ be an artinian ring and $J$ be its Jacobson radical. Consider the following two conditions:

(1) Every indecomposable injective left $R$-module is uniserial.

(2) $R$ is right serial.

Hill [4] proved that (1) and (2) are equivalent in case $R / J$ is a direct product of matrix rings over fields. Hill conjectured that (1) and (2) are equivalent for any artinian ring. Xue [5] answered the conjecture in the negative by providing counterexamples of local artinian rings, for which (1) and (2) are not equivalent. However, in this note we prove the equivalence of the two conditions in case each simple component of $R / J$ is finite dimensional over its center, thereby answering a question raised by Hill [4]. For any ring $S, Z(S)$ denotes the center of $S$, and if $D$ is a division subring of $S$, then $[S: D]_{r}\left([S: D]_{1}\right)$ denotes the right (left) dimension of $S$ over $D$. For any module $M, E(M)$ denotes the injective hull of $M$, and if $M$ has a composition series, $c(M)$ denotes the composition length of $M$. For basic concepts on rings and modules, we refer to [1].

We start with the following

Proposition 1. Let $D$ be a division ring finite dimensional over its center $F$ and for some positive integer $n$, let $D^{\prime}$ be a subdivision ring of $D^{n \times n}$ such that $D^{n \times n} e_{11}$ is a one-dimensional left vector space over $D^{\prime}$. Then $e_{11} D^{n \times n}$ is a one-dimensional right vector space over $D^{\prime}$.

Proof. Consider the subring $S$ of $D^{n \times n}$ generated by $D^{\prime} \cup F$. Then $S$ is a simple artinian ring, finite dimensional over $F$. Let $K=Z(S)$ and $K^{\prime}=Z\left(D^{\prime}\right)$. By Herstein [3, Theorem (4.1.1)] $D^{\prime} \otimes_{K^{\prime}} K$ is simple. As $S$ is a homomorphic image of $D^{\prime} \otimes_{K^{\prime}} K, S \approx D^{\prime} \otimes_{K^{\prime}} K$. Consequently $\left[D^{\prime}: K^{\prime}\right]=\left[D^{\prime} \otimes_{K^{\prime}} K: K\right]=[S:$ $K]=t<\infty$. Then $\left[D^{n \times n}: K\right]_{1}=n t$. As $F \subseteq K,\left[D^{n \times n}: K\right]_{1}=\left[D^{n \times n}: K\right]_{r}$. Then $\left[D^{n \times n}: K^{\prime}\right]_{1}=\left[D^{n \times n}: K\right]_{1}\left[K: K^{\prime}\right]$ yields $\left[D^{n \times n}: K^{\prime}\right]_{1}=\left[D^{n \times n}: K^{\prime}\right]_{r}$, so

Received by the editors December 14, 1995 and, in revised form, February 22, 1996.

1991 Mathematics Subject Classification. Primary 16P20; Secondary 16D50.

This research was partially supported by the Kuwait University Research Grant No. SM126.

(C)1997 American Mathematical Society 
that $\left[D^{n \times n}: D^{\prime}\right]_{1}=n=\left[D^{n \times n}: D^{\prime}\right]_{r}$ and hence $\left[e_{11} D^{n \times n}: D^{\prime}\right]_{r}=1$. This proves the result.

We now prove the following, which answers a question raised by Hill [4, p. 805]. The arguments used here are significantly different from those used by Hill [4].

Theorem 1. Let $R$ be an artinian ring such that $R / J$ is a direct product of matrix rings over finite-dimensional division rings. Then the following are equivalent:

(1) Every indecomposable injective left $R$-module is uniserial.

(2) $R$ is right serial.

Proof. Any artinian ring $R$ satisfies (1) ((2)) if and only if $R / J^{2}$ satisfies (1) ((2)) if and only if the basic ring $S$ of $R$ satisfies (1) ((2)). So without loss of generality, we take $R$ to be a basic ring with $J^{2}=0$. Let $T$ be a maximal orthogonal set of indecomposable idempotents in $R$.

Let every indecomposable injective left $R$-module be uniserial. Consider an $e \in T$, such that $e J \neq 0$. There exists unique $f \in T$, such that $e J f \neq 0$. Then $e J f=e J, V=e J f$ is a $\left(D, D^{\prime}\right)$-bivector space, where $D=e R e / e J e$ and $D^{\prime}=$ $f R f / f J f$. Let $E=E\left({ }_{R} D\right)$. Then $E / \operatorname{soc}(E) \approx R f / J f$ and $W=\operatorname{Hom}\left({ }_{D} V, D\right)=$ $\operatorname{Hom}\left({ }_{R} V, E\right) \approx R f / J f$. This gives $\operatorname{dim}\left({ }_{D^{\prime}} W\right)=1$. Further, $\operatorname{dim}\left({ }_{D} V\right)=n=$ $c\left({ }_{R} V\right)$ gives $\operatorname{dim}\left(W_{D}\right)=n$. We get an embedding $\mu: D^{\prime} \rightarrow D^{n \times n}$, such that ${ }_{D^{\prime}} W_{D} \approx \mu(d)\left(D^{n \times n} e_{11}\right)_{D}$. By Proposition $1, e_{11} D^{n \times n}$ is a one-dimensional right $\mu(D)$-vector space. However, ${ }_{D} V_{D^{\prime}} \approx \operatorname{Hom}\left(W_{D}, D\right) \approx{ }_{D}\left(e_{11} D^{n \times n}\right)_{\mu(D)}$. Consequently $\operatorname{dim}\left(V_{D^{\prime}}\right)=1$, i.e. $e J$ is simple. Hence $R$ is right serial.

Conversely, let $R$ be right serial. Let $E$ be an indecomposable injective left $R$ module, such that $\operatorname{soc}(E) \neq E$. We get an $e \in T$, such that $e J=R e J$ and $\operatorname{soc}(E)$ embeds in $e J$. As $e J$ is simple, there exists unique $f \in T$, such that $e J f=e J$. By using the notations in the previous paragraph, we get $V=e J f$ is a $\left(D, D^{\prime}\right)$-bivector space, $\operatorname{dim}\left(V_{D^{\prime}}\right)=1, n=\operatorname{dim}\left({ }_{D} V\right)=c\left({ }_{R}(e J)\right)$. Now $E$ is the injective hull of ${ }_{R} D$, so for $W=\operatorname{Hom}\left({ }_{D} V, D\right), \operatorname{dim}\left(W_{D}\right)=n, \operatorname{dim}\left({ }_{D^{\prime}} W\right)=c(E / \operatorname{soc}(E))$. By using Proposition 1, we get $\operatorname{dim}\left(D^{\prime} W\right)=1$. Consequently $E / \operatorname{soc}(E)$ is simple, and hence $E$ is uniserial.

\section{REFERENCES}

1. F. W. Anderson and K. R. Fuller, Rings and Categories of Modules, Springer Verlag, 1974. MR 54:5281

2. K. R. Fuller, On indecomposable injectives over artinian rings, Pacific J. Math. 29 (1969), 115-135. MR 40:186

3. I. N. Herstein, Non-commutative Rings, The Carus Monograph Number 15, The Mathematical Association of America, 1968. MR 37:2790

4. D. A. Hill, Rings whose indecomposable injective modules are uniserial, Canad. J. Math. 34 (1982), 797-805. MR 84h:16016

5. Weimin Xue, Two examples of local artinian rings, Proc. Amer. Math. Soc. 107 (1989), 63-65. MR 90d:16017

Department of Mathematics and Computer Science, Kuwait University, P.O. Box 5969, Safat 13060, KUWAit

E-mail address: singh@math-1.sci.kuniv.edu.kw 